\title{
la nature de Disneyland Paris
}

\section{par Pierre Alphandéry}

INRA-ESR, unité STEPE, 65, bd de Brandebourg, 94205 Ivry cedex

On ne retient habituellement de Disneyland Paris (situé en fait dans la Brie, à $30 \mathrm{~km}$ de la capitale, et initialement appelé Euro-Disneyland) que la présence emblématique de son parc de loisir. Toutefois, celui-ci ne couvre que 56 hectares, et les deux phases suivantes, programmées d'ici 2017, ne devraient pas, si elles se réalisent un jour, porter cette entreprise à plus d'une centaine d'hectares. Or ce sont 1947 hectares $\left(1 / 5^{\mathrm{e}}\right.$ de la surface de Paris) qui ont été alloués par contrat en 1987 à la société américaine, à des conditions sur lesquelles on ne reviendra pas dans le cadre de cet article $(*)^{1}$. Cette surface devrait être principalement consacrée à des réalisations immobilières (hôtels, centres commerciaux, bureaux, résidences, équipements divers...), mais une grande partie des terrains n'a jusqu'à présent pas été construite car la « crise » de l'immobilier a fortement contrarié l'euphorie initiale des décideurs publics et privés responsables de l'opération.

Environ 600 ha ont toutefois été aménagés dans le cadre de la première phase ${ }^{2}$, parmi lesquels figurent des espaces "naturels » auxquels une surface non négligeable a été réservée. Disneyland Paris, à l'image de ce que la société mère a accompli dans ses autres parcs, accorde en effet une attention particulière à ce qui est qualifié de " paysagement». Cela signifie non seulement que les infrastructures diverses ont été entourées d'un décor végétal (gazons, talus, plantations) particulièrement soigné, mais encore que l'ensemble du site a vu son paysage refaçonné, en particulier pour produire artificiellement et sans paysans une «nature » qui a pris place parmi les «attractions » du site. Les dirigeants de l'entreprise n'en font pas mystère, il s'agit d'inciter les visiteurs à prolonger leur séjour au maximum en élargissant toujours plus le spectre de l'offre de loisirs. C'est non seulement aux caractéristiques de cette nature artefact (fabriquée comme un objet), élément constitutif d'un décor et d'un imaginaire propres à Disney, que nous nous intéresserons, mais aussi à la dynamique dont elle est issue. Car ce véritable produit d'appel alliant fantaisie et hyperréalisme est aussi fondé sur la puissance économique et la maîtrise technique tout en s'inscrivant dans une conception radicale de l'aménagement de ce secteur de Marne-la-Vallée. Et cette nouvelle nature plantée dans une ville nouvelle est le produit d'un mouvement de concentration des activités économiques dont les ressorts sont la vitesse et le marché.

\section{Quand le faux donne plus que le vrai}

Rien n'existait avant Euro-Disneyland, selon ses dirigeants, qui désignaient en 1992 l'espace réaménagé comme un «monde entièrement nouveau surgi là où il n'y avait que des plaines mornes et plates $»^{3}$. Ces expressions ne doivent rien au hasard car il s'agissait bien de fabriquer un nouveau paysage urbain et naturel faisant table rase de celui qui le précédait. La quarantaine d'exploitants agricoles qui occupait les lieux a ainsi été expropriée pour céder la place aux « camemberts », conformément à

(*) Les notes sont reportées en fin d'article.

Ce texte est paru dans Natures extrêmes, n61 de Communications (1996), publié le Centre d'études transdisciplinaires (sociologie, anthropologie, histoire) - Ecole des hautes études en sciences sociales. II est repris avec l'aimable autorisation de la revue. 
l'ambitieux projet d'aménagement initial qui ne laissait plus subsister grand-chose des champs de betteraves ou de céréales et des bosquets qui composaient ce morceau de Brie.

D'un côté, des habitants et des associations, souvent animés par des écologistes, s'opposèrent avec énergie à ce qu'ils estimaient être un bouleversement radical imposé et largement immaîtrisé de leur territoire. De l'autre, au contraire, diverses personnes, dont beaucoup d'élus locaux, considéraient que de tels projets étaient inévitables dans une campagne proche de Paris et qu'il fallait n'en retenir que les retombées économiques annoncées. Ainsi, la critique et la lutte contre un projet porté à bout de bras par les pouvoirs publics ne furent pas, localement, partagées par tout le monde et, avant sa réalisation, l'idée même de la disparition programmée de ce qui caractérisait ce territoire rural avait fait son chemin dans bien des esprits. En témoigne cette déclaration d'un agriculteur, rapportée par l'Express en 1986 : « A 35 km de Paris, on ne peut plus être épargné par les mutations. Alors, voir mes terres grignotées par des villes nouvelles qui peuvent apporter des loubards en plus du béton ? J'aimerais mieux un Disneyland propre et net. »

La fabrication du «nouveau monde » a toutefois été rendue plus chaotique que prévue par les problèmes financiers rapidement rencontrés par l'entreprise franco-américaine. En effet, un an après une inauguration en grande pompe et objet d'une incroyable couverture médiatique, Euro-Disneyland se trouvait menacé de fermeture (risquant de se transformer ainsi en une friche d'un genre nouveau). Cette crise engendrait l'ajournement d'une partie des réalisations, produisant par là même un désordre paysager inattendu. S'il existe encore aujourd'hui des agriculteurs sur le site, leur maintien ne résulte donc pas d'un "Larzac " périurbain qui aurait fait reculer in extremis /es aménageurs. Dans les zones d'« aménagement différé », les exploitants restants ou avoisinants cultivent encore avec un bail annuel des terres qu'ils avaient été contraints de vendre, comme des jardiniers en sursis, survivants d'une époque révolue. Enfin, d'autres surfaces, demeurées sans affectation, constituent les images d'un laisser-aller (ou d'un retour au naturel sauvage) peu goûté chez Disney.

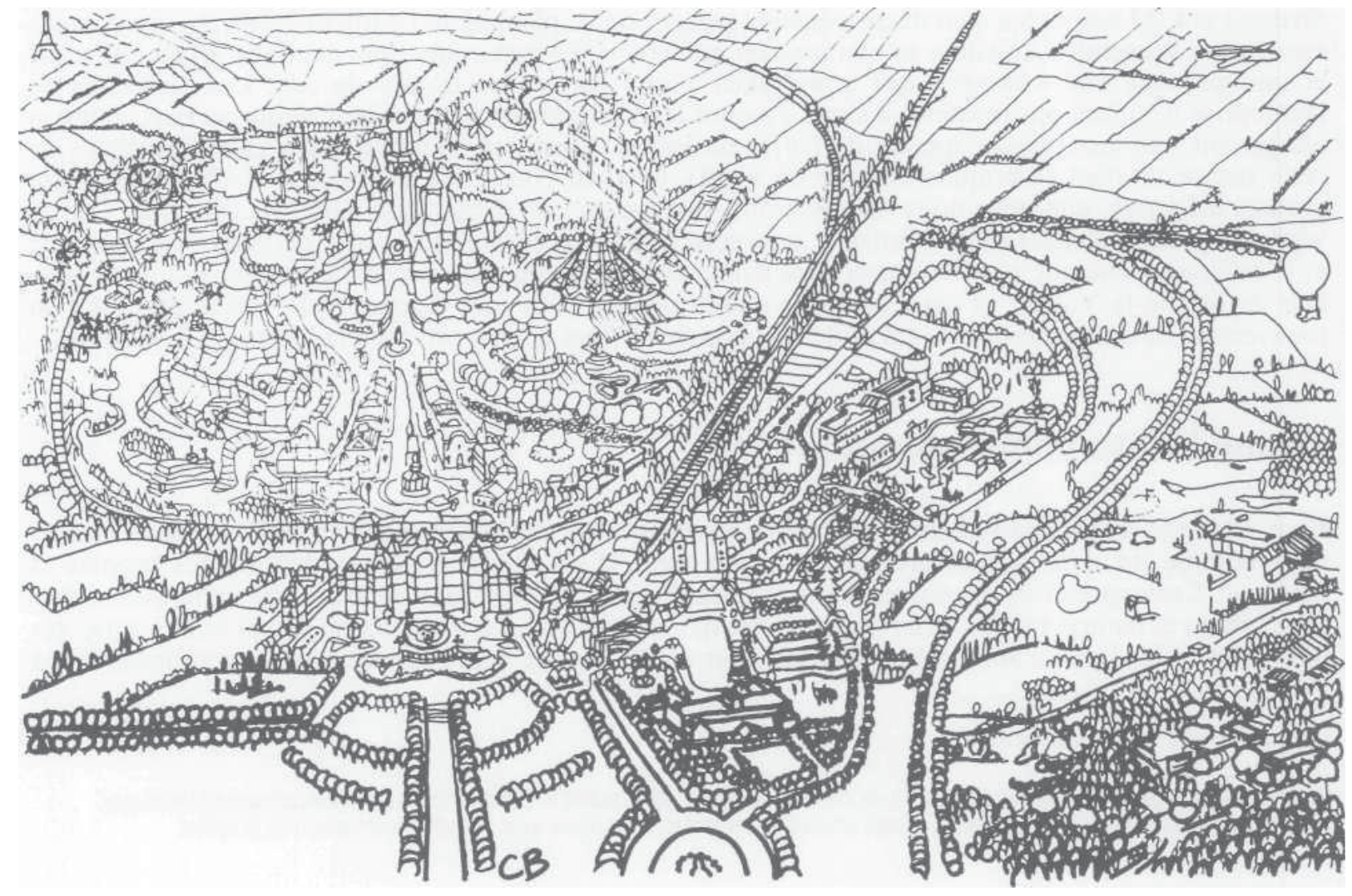


Il reste que les 600 ha déjà aménagés ont vu leur topographie entièrement refaçonnée : on y a créé des reliefs, des plans d'eau, des pelouses et de nouveaux lieux boisés. On a planté 360000 arbres et arbustes d'espèces importées du monde entier, fait venir des bisons, coulé $68000 \mathrm{~m}^{3}$ de rochers artificiels en béton armé. Et 420 personnes employées travaillent à temps plein sur l'ensemble du complexe de loisirs, à l'entretien des espaces verts ainsi fabriqués. On le voit, l'une des caractéristiques de Disneyland Paris réside dans le déploiement, pour sa réalisation, de moyens techniques puissants et sophistiqués qui n'ont pas manqué de frapper visiteurs et observateurs. Il faut rentrer dans ces lieux comme au sein d'un décor ou d'un haut lieu du faux car, comme l'expliquait Umberto Eco à propos des Disneyland américains, "Une fois que "le tout faux" est admis, il faut, pour qu'il soit apprécié, qu'il soit pris pour du vrai » ${ }^{6}$. Une carte Michelin spécialement éditée à l'occasion de l'ouverture du parc, le 12 avril 1992, a d'ores et déjà officialisé et comme authentifié l'existence des espaces refaçonnés. Pour réaliser ce que la publicité présente comme " un rêve devenu réalité », l'arrachement à la nature préexistante a donc été total et le "monde nouveau » a été fabriqué en rupture avec l'espace qui l'environne, comme un véritable isolat cultivant l'étrange et la fantaisie, tout en étant composé de décors évoquant des références culturelles qui se veulent familières aux visiteurs. Pris en charge par une organisation sophistiquée qui les délivre des soucis du monde quotidien, ces derniers se trouvent ainsi dépaysés au sens propre tout en se mouvant dans un univers connu et rassurant. Cette confrontation de sentiments opposés est redoublée par la coexistence d'infrastructures surdimensionnées qui desservent les lieux et d'attractions diverses fondées sur une miniaturisation du monde. Dans ce cadre, la nature fabriquée apparaît sous différentes formes complémentaires les unes des autres : elle constitue tantôt un écrin de verdure, tantôt un écran séparateur du monde extérieur, tantôt un décor sophistiqué.

Ecrin de verdure omniprésent, le gazon et les plates-bandes qui accompagnent partout les bâtiments et les routes doivent être propres et nets en permanence, comme un intérieur domestique ou un jardin privé. C'est pourquoi on y voit s'activer des armées de « nettoyeurs » en tout genre. Pas un brin d'herbe ne dépasse grâce à une noria d'hommes chevauchant partout des tondeuses automotrices qui, dans ce décor, ont remplacé les tracteurs des agriculteurs ${ }^{7}$. Le réseau des routes, des rocades, des parkings et des bâtiments nécessaires au fonctionnement de ce parc de divertissement s'affiche fièrement comme un signe de réussite de l'entreprise. Mais il est dans le même temps euphémisé par les espaces verts qui l'entourent, renvoyant le visiteur à un cadre plus intime. 50000 arbres et arbustes plantés sur des remblais de $10 \mathrm{~m}$ de haut séparent le parc à thèmes des zones environnantes de Marne-la-Vallée et du secteur des hôtels. Ils servent ainsi d'écran par rapport au monde extérieur et délimitent les contours de cette cité idéale dont s'inspirent peu ou prou tous les parcs de Disney. Dans le cas qui nous occupe, cette dernière est composée d'une série de décors hyperréalistes, mondes miniatures qu'accompagne chaque fois une végétation particulière, et cadres d'attractions consacrées aussi bien à l'Ouest mythique américain qu'aux contes de l'enfance ou aux conquêtes scientifiques de l'avenir. Ainsi, à Frontierland, pins sylvestres, arbres de Judée et cactus rappellent l'atmosphère du Colorado et les paysages de î'Arizona. A Fantasy-Land, 2,5 km de haies naturelles forment le labyrinthe d'Alice et à Discoveryland séquoias et araucarias évoquent les mystères et les secrets du futur. Enfin, dernière venue parmi les attractions, "Space Mountain » est tout entière consacrée au thème du décollage vers la lune ; on peut ainsi s'arracher à la terre et laisser derrière soi toute forme de végétation.

Le guide distribué à l'entrée du parc d'attractions détaille ce que les visiteurs peuvent espérer trouver dans son environnement immédiat. Si on laisse de côté le golf de 60 ha, le nouveau décor « naturel » créé est un paysage importé (mais bien connu des Français et Européens) : il doit en effet évoquer les grands espaces qui ont marqué l'histoire des USA. Et cette nature mythique américaine prend place aux côtés de l'exposition des marchandises du monde qu'offre en abondance le parc à ceux qui le fréquentent. Du même coup, le touriste peut ainsi déambuler dans le « grand magasin de l'humanité » en contemplant les signes évoquant la nature la plus grandiose : ainsi, grâce à la technique, « tout advient sur le mode de la disponibilité ${ }^{8}$. Mais cette disponibilité ne deviendra effective qu'en rencontrant celle du visiteur-consommateur des lieux. Car l'entrée dans le monde miniature de Disney nécessite une opération mentale qu'il n'est pas donné à tout le monde de réussir : refouler de sa mémoire les images de l'environnement immédiat qu'il a fallu au préalable traverser. Faute de quoi on ne retiendrait que l'énorme enchevêtrement des infrastructures de circulation composées d'échangeurs, de routes et de parkings, sas obligatoires qui n'incitent guère à l'émerveillement. Confronté à une signalétique moderne déclinant les hôtels, les points d'information et les gares, le visiteur aura en outre cherché une place pour sa voiture et affronté les multiples files d'attente. Les élus, étonnamment nombreux, sont ceux que ces épreuves n'arrêtent pas et qui en sortent mêmes confortés pour se couler dans le monde imaginaire délocalisé fabriqué par Disneyland Paris. 
Certains observateurs ont souligné que l'attachement à ces lieux (où l'on entre avec une réplique de passeport) résultait d'une part du changement d'échelle et, d'autre part, de la volonté de réconciliation générale qui les habitait ${ }^{9}$. Chez Disney, tout ce qui oppose ou inquiète est gommé : les cultures et les générations s'y côtoient sans heurt, le passé et le futur s'y succèdent sans rupture. Tout incite à disposer des éléments qui composent ce monde peu ordinaire et, dans un climat d'euphorie consumériste, à vivre une expérience partagée par des millions de gens venus de très loin. Mais seule une minorité pourra, après la visite du parc, aller se reposer dans des hôtels immergés au sein d'un décor naturel évoquant les paysages américains les plus connus et s'y retrouver, par les vertus conjuguées du marketing et de l'imaginaire, comme dans une résidence secondaire provisoire.

\section{L'appel de la forêt}

Parmi les hôtels destinés aux visiteurs fortunés, le Séquoia Lodge jouit d'un décor pour lequel ont été implantés 500 séquoias destinés à recréer la végétation du célèbre parc des Montagnes rocheuses. Selon le guide officiel, cet hôtel s'élève en outre « au bord du lac Buena Vista, environné par une superbe forêt de conifères à bois rouge. Une forêt comprenant plus de 2000 arbres a été plantée dans ce refuge des amoureux de la nature. Son architecture tout en bois et pierre évoque les chalets des parcs nationaux américains. D'un séjour au Séquoia Lodge vous emporterez le souvenir d'une promenade sur un sentier, du chant des oiseaux, ou l'image d'un eccureuil bondissant dans la mousse. La pierre brune et le bois rouge des murs, le vert-de-gris des toits sont en parfaite harmonie avec l'environnement ${ }^{10}$.

Le Journal du bois, quant à lui, émerveillé par l'oeuvre due à l'architecte français Antoine Grumbach, rapporte le rêve de ce dernier: « Planter une forêt au milieu de laquelle il installerait un hôtel merveilleux construit avec du bois, des pierres, du cuivre et des matériaux naturels [...]. Là où il n'y a pas si longtemps encore on ne trouvait que des champs de blé et de betterave, des aménagements paysagers hors du commun offrent aujourd'hui quatre hectares d'arbres de taille adulte plantés autour de l'hôtel sous les fenêtres panoramiques des chambres ${ }^{\mathrm{n}}{ }^{\text {. }}$.

Toutefois, les tarifs pratiqués à l'hôtel n'autorisent l'accès à cette nature qu'aux visiteurs fortunés (800 FrF par jour pour une chambre double en haute saison). Les autres peuvent toujours monter leur tente (l'emplacement vaut 300 FrF par jour) ou louer un bungalow au Ranch Davy Crockett (700 FrF par jour pour une cabane prévue pour deux adultes et deux enfants).

« A l'image des campements des pionniers de l'Ouest américain, le camp Davy Crockett est aménagé au coeur d'une forêt de 56 ha. Vous y avez rendez-vous avec la nature, dans un cadre qui rappelle le pays des trappeurs et des chercheurs d'or ${ }^{12}$.

La publicité n'omet pas de préciser que 110000 arbres ont été plantés en ce lieu, renouvelant totalement l'apparence de l'ancien bois. Au programme, promenades à pied, location de bicyclettes, feux de camp, visite d'une ferme reconstituée, etc. L'abondance des activités proposées transforme ainsi le cadre forestier en décor, prétexte à des activités familiales tournées vers la consommation de loisir.

Que représentent, alors, les multiples références du Guide et de la communication de Disney à un environnement harmonieux ou à la nature vierge rencontrée par les pionniers américains ? Comment oublier la forte densité des bungalows, la petitesse des emplacements de camping devant lesquels trônent des poubelles de plastique imitant des troncs d'arbres ou encore l'impossibilité de se promener ailleurs que sur quelques chemins où les bicyclettes se suivent en rangs serrés pour accéder aux boutiques du " campement » ? Manifestement, la forêt fabriquée ne fonctionne ici que comme décor soigné d'un jeu de rôle à grande échelle où les visiteurs-consommateurs constituent les figurants d'un véritable jardin d'acclimatation de la nature et de la culture américaines. Instrument de promotion d'un produit mis sur le marché (que les Anglo-Saxons qualifient du terme de resort). La fabrication de cette nature artefact (et qui se donne comme telle) produit du même coup les images symboliques auxquelles elle se rapporte. L'exemple de l'hôtel Séquoia Lodge, en particulier, qui constitue, nous dit la publicité, « comme un refuge au milieu de la forêt » renvoie à des références précises de la culture américaine. On pense notamment à Thoreau, écrivain philosophe et naturaliste, auteur de Walden ou la vie dans les bois et de La désobéissance civile, ouvrages qui marquent encore l'imaginaire politique et écologique des Etats-Unis et accordent au contact direct avec la nature des vertus morales et civiques. 


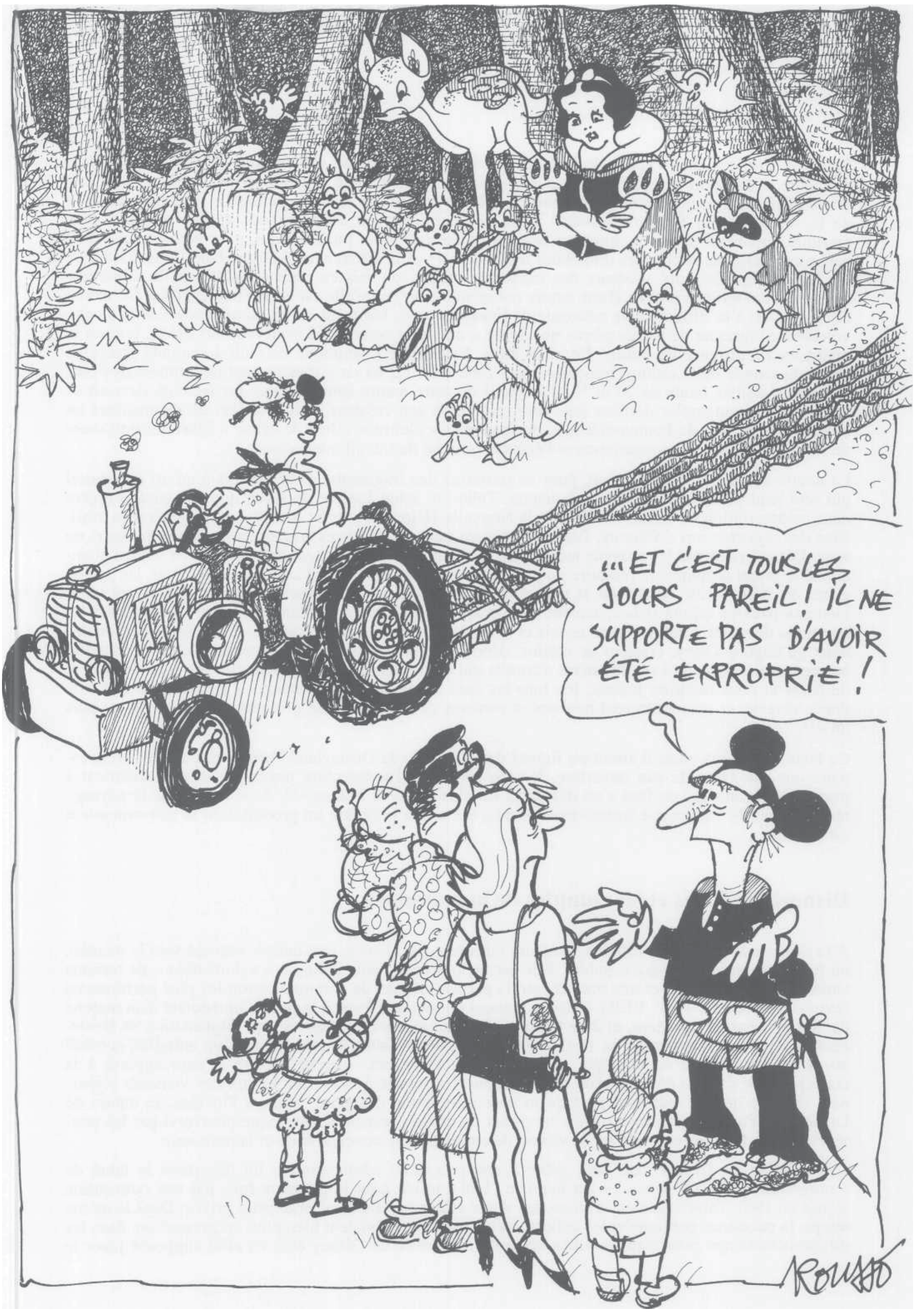


Sous les auspices de l'«appel de la forêt », Disneyland Paris se trouve ainsi être le milieu de la rencontre de la nature artefact et d'un tourisme soigneusement ciblé ${ }^{13}$. Mais ce paradoxe apparent estil très différent de celui relevé par Raphaël Larrère à propos des réserves naturelles instaurées au nom de la protection de l'environnement? Dans ces dernières, en effet, on use aussi d'artifices pour produire, mettre en scène et entretenir une nature destinée à un tourisme qui, pour pédagogique qu'il soit, n'engendre pas moins des retombées financières ${ }^{14}$. A la différence de Disneyland Paris, toutefois, les règles imposées aux visiteurs des espaces protégés, ou mis en réserves cultivent la " bonne » distance à conserver vis-à-vis d'une nature entretenue pour ce qu'elle est et non bouleversée. De même retrouve-t-on à la Bibliothèque nationale de France, dont le bâtiment est en cours d'achèvement, cette volonté de fabriquer de toutes pièces un espace " naturel » comme s'il avait toujours été là, tout en le maintenant à distance du public. En plein Paris, l'architecte Dominique Perrault a souhaité placer au coeur du temple de la culture francaise une " forêt " de 1,2 ha en y transportant notamment des pins sylvestres adultes hauts de $20 \mathrm{~m}^{15}$ ! L'objectif de cette nature-symbole, que les lecteurs devront se contenter de contempler derrière une vitre, est, selon son créateur, tout à la fois de reconstituer un morceau de la forêt de Fontainebleau et de figurer un " cloître ", "lieu de calme à l'abri des nuisances de la ville » favorisant la méditation et l'épanouissement du travail intellectuel.

La nature ayant été exclue, il faut, pour en retrouver des fragments, avoir recours à un art du naturel qui seul peut produire l'illusion de la nature. Telle est, selon Larrère, la leçon que Rousseau suggère dans sa description du jardin de Julie de la Nouvelle Héloïse ${ }^{16}$. Notre monde urbain généralise ainsi, dans des registres fort différents, l'usage d'artifices pour produire ces fragments de nature porteurs de sens. Démultipliée par les moyens techniques, cette inclination n'est toutefois pas neuve. Ayant abandonné le projet utopique de produire un paysage harmonieux en accord avec le corps social, les paysagistes du XIX ${ }^{\mathrm{e}}$ siècle ont conçu et réalisé des jardins urbains d'agrément, lesquels s'adressant aussi bien aux pauvres qu'aux riches, avaient une vocation hygiénique et philanthropique ${ }^{17}$. Les classes laborieuses devaient y oublier leurs soucis et trouver une opportunité de régénération physique dans les lieux qu'Eugène Derry, créateur de jardins, décrivait ainsi en 1893 : " Les grottes, rochers, cascades, barrages, ruisseaux, lacs seront autant d'attraits qui bien souvent nous rappelleront les doux souvenirs du passé et nous rendront joyeux. Ici, tous les âges et tous les tempéraments y trouvent un motif de douce rêverie, et tous rentreront heureux et contents de leur sortie, se promettant d'y revenir au plus tôt ${ }^{18}$.

Ce texte a cent ans, mais il aurait pu figurer dans le guide de Disneyland Paris dont bien des observateurs ont loué, lors de son ouverture, le soin apporté à l'architecture paysagère et l'acharnement à planter des arbres qui en font « un des parcs les plus boisé de France " ${ }^{\circ}$. A ceci près que le paysage redessiné par la compagnie franco-américaine a été conçu pour être un produit dont la consommation est payante.

\section{Disneyland Paris et les comptes de la puissance}

A la différence des jardins urbains, la nature fabriquée par Disney, tout entière tournée vars le marché, ne fait plus partie de l'espace public. Elle est incluse dans l'entreprise de "valorisation » de terrains situés tout près de Paris et très bien desservis par les moyens de communication les plus performants (avions, autoroutes, TGV, RER). Ceux-ci mettent Disneyland Paris à la portée immédiate d'un marché de 300 millions d'Européens, et il ne faut pas chercher ailleurs la raison de son implantation en Ile-deFrance, comme le confirmait en 1992 le PDG de la Walt Disney Company dans un entretien accordé au Monde ${ }^{20}$. L'enjeu économique représenté par cet énorme marché justifie le soin apporté à la conception de chaque élément d'un décor " naturel » destiné à mobiliser le flux des visiteurs séjournant dans ce qui se veut bien plus qu'un parc d'attraction ordinaire. Mais, à l'inverse, la nature de Disneyland Paris n'existe qu'à travers un projet de développement économique poursuivi par les pouvoirs publics français et fondé sur la vitesse de circulation, la concentration et la puissance.

L'installation de Disney s'est faite grâce à une procédure administrative lui décernant le label de " programme d'intérêt général » par laquelle l'Etat confiait pour la première fois, par une convention signée en 1987, l'aménagement d'un secteur d'une ville nouvelle à une entreprise privée. Dans le même temps, la puissance publique et les collectivités locales investissaient bien plus, en proportion, dans les infrastructures que pour d'autres villes nouvelles. L'arrivée de Disney était en effet supposée jouer le 
rôle de catalyseur d'un vaste projet concernant l'Est parisien auquel souscrivaient tant la majorité de droite du conseil régional et du conseil général de Seine-et-Marne que la gauche socialiste alors au pouvoir. Selon Michel Giraud, président de la région Ile-de-France, avec la création de Disneyland « c'est la réalité du plus grand axe tertiaire qui prend corps, de la Défenseà Marne-la-Vallée, qui relie l'ouest et l'est pour faire de la région capitale la métropole la plus performante d'Europe ${ }^{21}$.

On comprend mieux ainsi pourquoi le périmètre de Marne-la-Vallée qui a accueilli Disney s'appelle aujourd'hui le « Val d'Europe ». Vitrine technologique et touristique, Disneyland Paris était censé apporter une dynamique à un dessein continental dont rêvent encore beaucoup de nos décideurs. Cela fait de Mickey un personnage central aussi bien des « contes" que des « comptes de la puissance " 22 . Mais également de ses mécomptes. Car les récentes difficultés financières ont failli, on le sait, engendrer la fermeture de Disneyland Paris et ont provoqué l'ajournement des phases suivantes du projet. Le volontarisme et l'optimisme étaient fondés sur la conviction que le complexe touristico-immobilier agirait comme un aimant attirant de multiples autres activités tertiaires, intéressées, elles aussi, par le réseau de communication mis en place. Jean Poulit, directeur de l'établissement public de Marne-laVallée et l'un des principaux promoteurs de la venue de Disney dans la Brie, affirmait ainsi en 1991 : « Le coeur du dispositif sera l'interconnexion des TGV avec côte à côte la gare du TGV et la gare terminale de la ligne A du RER. C'est là que se trouve le coeur de la richesse de Marne-la-Vallée, celle qui, effectivement, poussera les visiteurs à venir nombreux nous rejoindre, mais également les chefs d'entreprise à s'implanter massivement dans l'Est parisien ${ }^{23}$.

On ne saurait mieux illustrer les liens qu'entretiennent la puissance économique et la vitesse, phénomène déjà analysé par Marx qui, selon Lothar Baier, avait montré, dans les fondements de la critique de l'économie politique, comment l'extension planétaire du marché débouche sur la « destruction de l'espace grâce au temps ».

« L'espace, écrit à son tour Lothar Baier, ne possède donc plus aucune dimension qui lui soit propre et n'offre plus par lui-même aucune résistance matérielle : il n'apparaît plus que comme facteur du temps qui absorbe toutes les propriétés de l'espace " ${ }^{24}$.

C'est bien ainsi qu'apparaît l'espace investi par Disneyland Paris. Objet permanent du discours de la performance technique et économique, il n'est plus évoqué que sous forme de ratios : ne comptent désormais que le nombre des visiteurs du parc, l'argent qu'ils dépensent, la rentabilité des investissements et les retombées financières qu'il occasionne. Ayant ainsi perdu ses caractéristiques physique et anthropologique initiales, le secteur 4 de Marne-la Vallée, transformé en un gigantesque décor avec lequel les autochtones n'ont plus de liens, s'apparente à ces "non-lieux » voués à la circulation et à la consommation qu'évoque Marc Auge. Les cinq communes « accueillant » Disneyland Paris, riches et suréquipées grâce aux impôts locaux, n'ont pas pour autant vu arriver les autres activités économiques ou culturelles espérées qui leur permettraient d'être autre chose que des Disney-villes ${ }^{25}$. La transformation rapide de ces bourgs ruraux est emblématique de ce qui se passe en Ile-de-France, et particulièrement en Seine-et-Marne. Selon un document du conseil général de ce département, chaque année 1000 ha d'espaces naturels et agricoles sont consommés (sans même compter Disneyland) par l'urbanisation, et les trois quarts des constructions nouvelles sont situées dans la partie nord-ouest ${ }^{26}$. Des enquêtes montrent que même les communes qui cherchent à préserver leur vocation agricole se voient souvent contraintes d'accepter la construction de nouveaux logements sous la pression des pouvoirs publics. Ces derniers cherchent en effet à « rentabiliser » les infrastructures de transport réalisées et à répartir la croissance urbaine. Ainsi, la commune de Jossigny, coincée entre Disneyland et des villes en pleine expansion, est-elle tenue de démontrer l'intérêt pédagogique et écologique qu'aurait pour ses voisines la préservation d'un tissu d'exploitations pour espérer conserver une partie de son territoire agricole. Mais, dans le même temps, personne ne semble en mesure de maîtriser les déséquilibres multiples engendrés par le développement anarchique que connaît la Seine-et-Marne, département qui présente la plus forte croissance démographique de France : 2,68\% de progression annuelle de 1982 à 1990 contre 0,53\% pour la France entière durant la même période.

Dans ce contexte, le nouveau paysage " inventé » par Disney est révélateur d'un double mouvement : tout à la fois producteur et produit de la concentration urbaine, Disneyland absorbe l'espace rural et s'applique à redessiner, dans les interstices des constructions, une nature ludique où le faux s'affiche sans scrupule à travers l'évocation du passé. Cette nature péri-urbaine s'inscrit en contrepoint de celle que les communes du "rural profond » tentent aujourd'hui, en France désespérément de valoriser. « Les primevères et les paysages ont un défaut grave : ils sont gratuits. L'amour de la nature ne 
fournit de travail à nulle usine ", se désolait déjà un personnage du Meilleur des mondes, d'Aldous Huxley ${ }^{27}$. Et il expliquait les remèdes envisagés : envoyer les masses à la campagne pour pratiquer des sports consommant des articles manufacturés et du transport. A l'évidence, la place prise par ce type de dépenses dans notre société montre que la solution imaginée par Huxley était prémonitoire. A Disneyland Paris, les aménagements réalisés ont fait mieux encore : après avoir éliminé les agriculteurs, on a en effet refabriqué de la nature en fonction de loisirs qui induisent eux-mêmes des dépenses de transport et de logement ainsi que de consommation en tout genre.

Disneyland Paris est unique, et le restera sans doute longtemps, par sa dimension spatiale et financière, la sophistication technique de son «industrie du faux » et l'ampleur des investissements publics qui y ont été effectués. Mais la production de nature sans paysans semble, quant à elle, promise à un bel avenir, que ce soit dans les parcs situés en zone péri-urbaine ou dans les espaces protégés et mis en réserve des zones rurales. On peut aussi imaginer que cette production de nature subira la concurrence des mondes virtuels auxquels nous sommes, paraît-il, promis. Dans ce dernier cas, il ne sera même plus nécessaire de se déplacer et l'on pourra profiter " en temps réel », chez soi ou dans un musée, des primevères et des paysages évoqués par Huxley •

\section{Notes}

1. Voir ALPHANDERY P., 1993. L'insoutenable développement d'Euro-Disneyland. Ecologie politique, 5, 51-76.

2. On y trouve le parc à thèmes comportant 30 attractions, 6 hôtels représentant au total 5200 chambres, un centre de divertissements de $18000 \mathrm{~m}^{2}$ carrés (avec 6 magasins, 5 restaurants, 2 bars,

1 discothèque, 1 dîner-spectacle sur le thème de Buffalo Bill), un grand parking, le ranch Davy Crockett, un lac de 4 ha et un golf de 18 trous. Enfin, une gare accueille le TGV et le RER, dont la ligne a été prolongée spécialement de $10 \mathrm{~km}$.

3. Expression utilisée par P. Fitzpatrick, PDG d'Euro-Disney SCA, au cours d'un entretien avec J.-P. Elkabbach, le 15 avril 1992 sur Europe 1.

4. Nom attribué au personnel de Disney (les cast members) par les « indigènes ".

5. L'Express du 21-27 mars 1986.

6. Eco U., 1993, La guerre du faux, Grasset, Paris.

7. Mais parfois les manifestations paysannes sont l'occasion du retour des occupants précédents, qui viennent bloquer les routes donnant accès au parc. Durant les négociations de la Politique agricole commune de juin 1992, Disneyland a ainsi été érigé en symbole de la menace américaine à l'encontre de l'agriculture française.

8. Finkielkraut A., 1991. Le Mécontemporain, Gallimard, Paris, p. 179.

9. BRUCKNER P., 1995. La tentation de l'innocence, Grasset, Paris. 10. EURo DisNey Resort, 1992. le Guide, p. 132.

11 Han F., 1993. Le bois chez Mickey et oncle Picsou. Journal du bois, mai-juin 1993. p. 48

12. EURO DiSNEY RESORT, 1992. le Guide, p. 144.

13. On peut observer ailleurs ce phénomène, notamment dans les Center Parcs, présents en Normandie et en Sologne. Ces vastes domaines offrent aux visiteurs une bulle de nature tropicale aquatique et des « cottages » à louer « au coeur de la forêt ».
14. LARRERE R., 1994. L'art de produire la nature. Une leçon de Rousseau. Le courrier de l'environnement de l'TNRA, 22, 5-13.

15. NOUGAREDE O., ALPHANDERY P., 1995. Le cloître forestier de la Bibliothèque nationale de France. Arbre actuel, avril-mai 1995.

16. LARRERE R., op. cit.

17. Voir Lugrnbul.h Y., 1989. Paysages. La Manufacture, Lyon.

18. DERRY E., 1893. Histoire générale des jardins. Cité par LUGinBulH Y., op. cit. p. 109.

19. LANQUAR R., 1992. L'empire Disney, PUF « Que sais-je? ", Paris, p. 116.

20. $\ll$ Un énorme bassin de population habite à moins de deux heures de voiture du parc et 300 millions de gens vivent à moins de deux heures d'avion » (Le Monde des 4-5 octobre 1992).

21. Entretien avec Michel Giraud. 5 puissance IV (journal du Syndicat d'agglomération nouvelle des portes de la Brie), 5 octobre 1991.

22. Nous paraphrasons ici le titre d'un livre relatant l'histoire et les enjeux de la comptabilité nationale et du Plan en France. Voir FOURQUET F., 1980. Les comptes de la puissance, Encres, Editions Recherches.

23. Poult J., 1991. Euro-Disney dans le cadre du développement urbain de Marne-la-Vallée. Actes des rencontres d'EPA France, 17 et 18 juin 1991, p. 243

24. BAIER L., 1981. Les Allemands maîtres du temps. La Découverte, Paris, p. 38.

25. Voir, par exemple : Les communes voisines de Disneyland Paris attendent l'essor promis, Le Monde du 13 avril 1995.

26. Conseil général de Seine-et-Marne, 1991. Charte départementale d'aménagement. p. 16.

27. HuXley A., 1970. Le Meilleur des mondes. Le livre de poche, Paris, p. 59. 